\title{
The solar system: A new geometric classification and prediction of a new asteroid
}

\author{
Ahmad Hazaymeh ${ }^{1}$, Mosab Hawarey ${ }^{2}$, Khaled Hazaymeh ${ }^{3}$ \\ ${ }^{1}$ Geodesic Dome Company, Astronomy and Mathematics Division, New York, NY 10001, USA \\ ${ }^{2}$ Geodesic Dome Company, Geodesy and Geophysics Division, New York, NY 10001, USA \\ ${ }^{3}$ University of Calgary, Department of Geomatics Engineering, Calgary, Alberta, Canada \\ Email Address: \\ info@geodesic-dome.biz (A. Hazaymeh)
}

\section{To cite this article:}

Ahmad Hazaymeh, Mosab Hawarey, Khaled Hazaymeh. The Solar System: A New Geometric Classification and Prediction of a New Asteroid. International Journal of Astrophysics and Space Science. Vol. 3, No. 2, 2015, pp. 16-19. doi: 10.11648/j.ijass.20150302.11

\begin{abstract}
One of the most popular theories of measuring planetary distances in the solar system is Bode's law, but this law has some deviations when compared to the real distances. In this paper a new classification (Hazaymeh-Hawarey Classification) is presented. This classification aims to explain the inability of Bode's law to identify the two orbital dimensions of Neptune and Pluto, and it provides a new geometric image for the orbital distribution of the solar system, including Neptune and Pluto. This new classification shows that orbits of the solar system are divided into four equal groups, where the $(10 / 3=4)$ is true. It also discovers that the two dimensions of Neptune and Pluto are much more systematic in the solar system, compared to Bode's law. The new classification includes the adjustment of the distances of Sedna and Eris, and enables the mathematical identification of a new asteroid located after Iris, even before the astronomical verification. The new classification reveals the symmetry and the anomaly of the solar system orbits and that the diameters of the first and fourth groups follow a geometric sequence. According to this, the diameter of the new asteroid can be determined.
\end{abstract}

Keywords: Bode's Law, Orbital Distances, New Geometry of the Solar System, New Astroid

\section{Introduction}

Bode's sequence, as in [1], gives good results with respect to the distance of the first eight orbits of the ten solar system orbits. It was reviewed and discussed by many researchers, as in [2], [3], and [4]. This sequence has three steps:

First: The orbits are given these numbers $(0,3,6,12,24$, 48, 96, 192, 384, and 768);

Second: Adding (4) to each number $(0+4),(3+4),(6+$ $4),(12+4),(24+4),(48+4),(96+4),(192+4),(384+$ $4)$, and $(768+4)$;

Third: Divide the outputs by $10: 4 / 10,7 / 10,10 / 10,16 / 10$, 28/10, 52/10, 100/10, 196/10, 388/10, and 772/10;

This gives: $(0.4,0.7,1.0,1.6,2.8,5.2,10,19.6,38.8$, and 77.2).

Nevertheless, Bode's law $\left(\mathrm{R}=(3) *(2)^{\wedge}(\mathrm{n}-2)+4\right)$, gives $0.55 \mathrm{AU}$ to Mercury:

$\left(\mathrm{R}^{*}\right)=(3) *(2)^{(1-2)}+4$

$\left(\mathrm{R}^{*}\right)=(3) *(2)^{(-1)}+4$;

$\left(\mathrm{R}^{*}\right)=(3) *(0.5)+4=5.5$;
$\mathrm{R}=\left(\mathrm{R}^{*}\right) / 10=5.5 / 10=0.55$.

Table 1 shows the differences between the orbital distances from the Sun in reality and according Bode's law.

Table 1. Real orbital distances from the Sun compared to distances according to Bode's law

\begin{tabular}{llll}
\hline Number & Orbit & $\begin{array}{l}\text { Real Distance } \\
(\mathbf{A U})\end{array}$ & $\begin{array}{l}\text { Distance according to } \\
\text { Bode's law (AU) }\end{array}$ \\
\hline 1 & Mercury & 0.3870 & 0.4 or 0.55 \\
2 & Venus & 0.7200 & 0.7 \\
3 & Earth & 1.0000 & 1 \\
4 & Mars & 1.5250 & 1.6 \\
5 & Asteroids & 2.7700 & 2.8 \\
6 & Jupiter & 5.2070 & 5.2 \\
7 & Saturn & 9.5450 & 10 \\
8 & Uranus & 19.2370 & 19.6 \\
9 & Neptune & 30.8600 & 38.8 \\
10 & Pluto & 39.5545 & 77.2 \\
\hline
\end{tabular}

\section{Proof that 10 Divided by 3 Equals 4}

It might seem impossible to divide ten orbits into three equal groups containing four orbits each. However, this will 
be true if we use the concept of the "Common Orbit".

\subsection{The Orbital Group and Common Orbit}

The orbital group is defined as the existents of four sequent orbits of the orbits of the solar system in an arithmetic sequence or a geometric sequence.

Whereas, the common orbit is defined as the existence of an orbit at the end of an orbital group, then it comes at the beginning of the following group. The common orbits are; Mars, Saturn and Pluto, if a fourth group exist. Table 2 shows the orbital groups and common orbits.

Table 2. The orbital group and common orbit

\begin{tabular}{llll}
\hline Number & $\mathbf{1}^{\text {st }}$ orbital group & $\mathbf{2}^{\text {nd }}$ orbital group & $\mathbf{3}^{\text {rd }}$ orbital group \\
\hline 1 & Mercury & Mars & Saturn \\
2 & Venus & Asteroids & Uranus \\
3 & Earth & Jupiter & Neptune \\
4 & Mars & Saturn & Pluto \\
\hline
\end{tabular}

\section{Calculations}

\subsection{The Control Sequence (Hazaymeh-Hawarey Classification)}

The sequence, which controls the dimensions of the solar system orbits from the sun, is:

$\mathrm{D}=\mathrm{O}^{1}(\mathrm{Z}-\mathrm{C})$

Where:

D: the distance of the orbit from the Sun;

$\mathrm{O}^{1}$ : the distance of the first orbit in each group;

$Z$ : the arithmetic sequence in the first and third group $(1,2$,

$3,4)$ and the geometric sequence in the second group $(1,2,4$, $8)$;

$\mathrm{C}$ : numerical anomaly, which is the decrease and increase in the number of orbit, in the orbital sequence.

\subsection{The Distances of the Orbital Groups from the Sun}

\subsubsection{The First Group}

It consists of the orbits of (Mercury, Venus, Earth, and Mars). The orbital dimensions of this group, as seen in Table 3, follow an arithmetical sequence $(1,2,3,4)$. But, the numerical anomaly is derived by dividing the true distance of the orbit by the distance of the first orbit in its group, and then the result is subtracted from the orbital number in the sequence. For example, the numerical anomaly of Venus orbit is $2-(0.7200 / 0.3870)=1.1395$.

Table 3. The astronomical unit of the orbital distance from the sun for the first group

\begin{tabular}{ll}
\hline The orbit & $\begin{array}{l}\text { The astronomical unit }(A U) \text { of the orbital distance } \\
\text { from the Sun }\end{array}$ \\
\hline Mercury & $0.3870 \times(1-0.0000)=0.3870$ \\
Venus & $0.3870 \times(2-0.1395)=0.7200$ \\
Earth & $0.3870 \times(3-0.4160)=1.0000$ \\
Mars & $0.3870 \times(4-0.0594)=1.5250$ \\
\hline
\end{tabular}

\subsubsection{The Second Group}

It consists of the orbits of (Mars, Asteroids belt, Jupiter and Saturn). The orbital distances of this group, as seen in Table 4, follow geometric sequence $(1,2,4,8)$. The numerical anomaly of this group is calculated as the first group calculation. For example: the numerical anomaly of asteroids belt orbit $=2-(2.7700 / 1.5250)=1.1836$.

Table 4. The astronomical unit of the orbital distance from the sun for the second group

\begin{tabular}{ll}
\hline The orbit & $\begin{array}{l}\text { The astronomical unit }(\mathrm{AU}) \text { of the orbital distance } \\
\text { from the Sun }\end{array}$ \\
\hline Mars & $1.5250 \times(1-0.0000)=1.5250$ \\
Asteroid & $1.5250 \times(2-0.1836)=2.7700$ \\
Jupiter & $1.5250 \times(4-0.5856)=5.2070$ \\
Saturn & $1.5250 \times(8-1.7410)=9.5450$ \\
\hline
\end{tabular}

\subsubsection{The Third Group}

It consists of the orbits of (Saturn, Uranus, Neptune, and Pluto). The orbital distances of this group, as seen in Table 5, follow an arithmetic sequence $(1,2,3,4)$. The numerical anomaly is derived by dividing the true orbital distance by the distance of the first orbit in its group, and then the number of the orbit in its group is subtracted from the result. For example: the numerical anomaly of Uranus orbit = $(19.2370 / 9.5450)-2=0.0154$.

Table 5. The astronomical unit of the orbital distance from the sun for the third group

\begin{tabular}{ll}
\hline The orbit & $\begin{array}{l}\text { The astronomical unit (AU) of the orbital distance } \\
\text { from the Sun }\end{array}$ \\
\hline Saturn & $9.5450 \times(1+0.0000)=9.5450$ \\
Uranus & $9.5450 \times(2+0.0154)=19.2370$ \\
Neptune & $9.5450 \times(3+0.2331)=30.8600$ \\
Pluto & $9.5450 \times(4+0.1442)=39.5545$ \\
\hline
\end{tabular}

The numerical anomalies in the numbers of the arithmetic sequence of this group are increased anomalies, while they are decreased anomalies in the previous two groups.

\subsection{Conclusions of Calculations}

A. The first orbital group in the Solar System (Mercury, Venus, Earth, and Mars) is controlled by an arithmetic sequence.

B. The second orbital group in the Solar System (Mars, Asteroids belt, Jupiter, and Saturn) is controlled by a geometric sequence.

C. The third orbital group in the Solar System (Saturn, Uranus, Neptune, and Pluto) is controlled by an arithmetic sequence.

D. Pluto shows discipline in its dimension from the sun. According to the new arithmetic sequence, where the increased numerical anomaly does not exceed $(0.1442)$, and Neptune does not exceed (0.2331). While the decreased numerical anomaly of Earth is (0.4160), while Saturn shows a decreased numerical anomaly of (1.7410) which means that its numerical anomaly is more than (10) times more than in Pluto.

E. The sequence of the second orbital group is a geometric sequence, while the sequence of the first and the third groups are arithmetic sequences. Thus, the second group is an 
incompatible group.

\section{Discussion}

\subsection{The Orbital Symmetry}

Orbital symmetry is the distance between two orbits in two orbital groups controlled by geometric sequence, such as $\left(10^{0}\right.$ and $\left.10^{2}\right)$ taking into account the existence of some numerical anomaly.

Is it probable that there is a fourth orbital group in the solar system?! If it exists, would it be controlled by a sequence that follows arithmetic or geometric sequence?!

\subsubsection{Fourth Group as an Arithmetic Sequence}

Assuming that the fourth orbital group is an arithmetic sequence, and it consists of four orbits. Accordingly, the first orbit of this group is Pluto. We will call the other orbits as the second orbit, the third orbit, and the fourth orbit, respectively.
Now let us assume that the numerical anomaly of each orbit is zero. Hence, the results will be as seen in Table 6 .

Table 6. The astronomical unit of the orbital distance from the sun for the fourth group

\begin{tabular}{ll}
\hline The orbit & $\begin{array}{l}\text { The astronomical unit (AU) of the orbit distance from } \\
\text { the Sun }\end{array}$ \\
\hline Pluto & $39.5545 \times(1-0)=39.5545$ \\
Second Orbit & $39.5545 \times(2-0)=79.1090$ \\
Third Orbit & $39.5545 \times(3-0)=118.6635$ \\
Fourth Orbit & $39.5545 \times(4-0)=158.2180$ \\
\hline
\end{tabular}

Based on these results, we will find that the dimensions of these orbits increase by more than (100) times over the first group. By adding an interesting factor through entering a numerical anomaly of the first orbital group to the orbital numbers of the fourth orbital group, we will have a marvelous result making us calling these orbits (the nadirs of the first orbital groups). Table 7 shows the nadirs of the first orbital groups.

Table 7. The nadirs of the first orbital group

\begin{tabular}{|c|c|c|c|}
\hline Description of the orbital distance & The orbital distance (AU) & Description of the ratio & The ratio \\
\hline $\begin{array}{l}\text { The distance of the orbit of Pluto } \\
\text { (which is Mercury nadir) }\end{array}$ & $39.5545(1-0)=39.5545$ & The ratio between Mercury and its nadir & $39.5545 / 0.3870=102.2080$ \\
\hline $\begin{array}{l}\text { The distance of the second orbit } \\
\text { (which is Venus nadir) }\end{array}$ & $39.5545(2-0.1395)=73.5911$ & The ratio between Venus and its nadir & $73.5911 / 0.7200=102.2099$ \\
\hline $\begin{array}{l}\text { The distance of the third orbit (which } \\
\text { is Earth nadir) }\end{array}$ & $39.5545(3-0.4160)=102.2088$ & The ratio between Earth and its nadir & $102.2088 / 1.0000=102.2088$ \\
\hline $\begin{array}{l}\text { The distance of the fourth orbit (which } \\
\text { is Mars nadir) }\end{array}$ & $39.5545(4-0.0594)=155.8685$ & The ratio between Mars and its nadir & $155.8685 / 1.5250=102.2089$ \\
\hline
\end{tabular}

\subsubsection{Fourth Group as a Geometric Sequence}

Assuming that the fourth orbital group is a geometric sequence as $(1,2,4,8)$. Then, here, we will find that the orbits, which have numbers of $(1,2,4)$ will be Mercury nadir (i.e. Pluto), Venus nadir (i.e. second orbit; unnamed), and Mars nadir (i.e. fourth orbit; unnamed). In addition a new orbit will enter in the sequence, where its number is (8), and its orbit is farer by more than (100) times away from the asteroids belt orbit. For this reason it will be given the same numerical anomaly of the asteroids belt orbit: $39.5545(8-0.1836)=309.1738$.

Also, the times ratio between the asteroids belt and its nadir: $309.1738 / 2.7700=111.6150$.

We note that the geometric sequence has caused two problems in the fourth orbital group:

A. The orbit, which is the Earth nadir, disappeared.

B. An imbalance of the times ratio between the fourth orbital group and the first orbital group, which is (102) times according to the arithmetic sequence, while it is (102) times in the geometric sequence for Mercury nadir, Venus nadir, and Mars nadir. However, it reaches (111.6150) times in the asteroids belt nadir.

\subsection{Other Nadirs}

Pluto is considered a dwarf plant of Kuiper belt that ranges from (30) $\mathrm{AU}$ to (55) AU, but its main part ranges from (39.5) to (48) AU. Comparing Mercury distance in reality (0.3870) AU to its distance according to Bode's law (0.55) AU, we will find that Kuiper belt is located at about more than (100) times from Mercury distance, this leads us to believe that all Kuiper belt, but not Pluto only, is the nadir of Mercury orbit. Therefore, if we want to find the orbits of Venus, Earth, and Mars nadirs, we must go after Kuiper belt at (73.5911) AU, (102.2088) AU, and (155.8685) AU.

Iris is the second dwarf planet in the solar system; it is located at about (100) AU, so it will be the Earth's nadir. The strange destiny in the word (Iris) is its similarity to the words $($ Ardh $=$ in Arabic) and (Ares = in Aramaic), and (Earth), which all mean the Earth planet.

Based on the previous discussion, there will be two undiscovered nadirs: they are Venus nadir, which is located at about (73.5911) AU, and Mars nadir, which is located at about (155.8685) AU.

\subsection{The Second Asteroid in the Fourth Group}

Sedna is an asteroid, and it was discovered in 2003, as in [5]. It is located at about (76.16) AU. If we know that Venus nadir in the sequence lies between (73.5911) $\mathrm{AU}$ and (79.1090) AU, then the asteroid (Sedna) will be in fact Venus nadir. 


\subsection{The Fourth Asteroid in the Fourth Group}

This asteroid is still undiscovered, but the sequence of the fourth group expects its existence at a distance of (155.8685) AU.

Can we expect something else about this asteroid?! The Answer is: Yes; we can expect its diameter.

By comparing the diameters of the first three planets (Mercury, Venus and Earth) which are in the first group, with the diameters of the first three asteroids (Pluto, Sedna, and Eris) which are in the fourth group and the nadirs of the first three planets, we will find that the ratio between the diameters of the planets of the first group and the diameters of asteroids of the fourth group follow a geometric sequence as following:

The ratio between the diameters of Mercury and its nadir (Pluto) is: $4889 \mathrm{~km} / 2300 \mathrm{~km}=2.12$

The ratio between the diameters of Venus and its nadir (Sedna) is: $12102 \mathrm{~km} / 1600 \mathrm{~km}=7.56$

Taking into account that the diameter of Sedna is less than $1600 \mathrm{~km}$, as in [6], if we knew that the standard ratio between Venus and its nadir is (1:8), then we will get a shorter length of Sedna's diameter as: $12102 \mathrm{~km} / 8=$ $1512.75 \mathrm{~km}$.

The ratio between the diameters of the Earth and its nadir (Iris) is: $12756 \mathrm{~km} / 3000 \mathrm{~km}=4.25$.

By focusing on these ratios, we will find that they are very close to the geometric sequence:

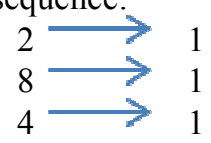

In addition, we note that there is abnormality in the order of the sequence; they should be like this:

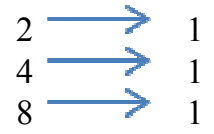

Accordingly, we can expect that the ratio between the diameters of Mars and its nadir will be the fourth amount in the previous geometric sequence. That means that the ratio between Mars and its nadir is (1:16). According to this, the diameter of Mars nadir will be: $6794 \mathrm{~km} / 16=425 \mathrm{~km}$.

If we want to broaden our expectation a little bit, we will assume that the ratio between the two diameters of Mars and its nadir is not exactly $(1: 16)$, but it may be lower (15.6) or higher (16.4). This thing will make Mars nadir locate between the two possibilities, which are: $6794 \mathrm{~km} / 15.6=$ $436 \mathrm{~km}$ and $6794 \mathrm{~km} / 16.4=414 \mathrm{~km}$.

According to this expectation, the diameter of Mars nadir will be $(425 \pm 11) \mathrm{km}$.

\section{Conclusions}

It seems that mathematics is still able to grant us a new astronomic knowledge that has not been discovered yet. Since Bode's law was able to predict Uranus planet 200 years ago, the new classification presented in this paper is able to discover new geometry of the distribution of solar system and it can predict new asteroid in the fourth group (with specified distance and specified diameter). This deserves more practical efforts to experimentally validate the existence of this asteroid astronomically, and explain the phenomenon physically.

\section{References}

[1] Nieto, M. (1972) The Titius-Bode law of planetary distances: its history and theory, $1^{\text {st }}$ ed. Oxford, UK: Pergamon Press.

[2] Graner, F. \& Dubrulle, B. (1994) Titius-Bode laws in the solar system I. Scale invariance explains everything. Astronomy \& Astrophysics, Vol. 282, No. 1, pp. 262-268.

[3] Hayes, W. \& Tremaine, S. (1998) Fitting selected random planetary systems to Titius-Bode laws. Icarus, Vol. 135, No. 2, pp. 549-557. DOI: 10.1006/icar.1998.5999.

[4] Lynch, P. (2003) On the significance of the Titius-Bode law for the distribution of the planets. Monthly Notice of the Royal Astronomical Society, Vol. 341, No. 4, pp. 1174-1178. DOI: 10.1046/j.1365-8711.2003.06492.x.

[5] Brown, M., Trujillo, C. \& Rabinowitz, D. (2004) Discovery of a Candidate Inner Oort Cloud Planetoid. Astrophysical Journal, Vol. 617, No. 1, pp. 645-649. DOI: 10.1086/422095.

[6] Stansberry, J., Grundy, W., Brown, M., Cruikshank, D., Spencer, J., Trilling, D. \& Margot, J.-L. (2007) 'Physical Properties of Kuiper Belt and Centaur Objects: Constraints from Spitzer Space Telescope' In: Barucci, M. A., Boehnhardt, H. \& Cruikshank, D. P. (eds.) The Solar System Beyond Neptune. University of Arizona Press, pp. 161-179. 